\title{
Longitudinal assessment of antibiotic resistance gene profiles from the infant gut microbiome
}

\section{CURRENT STATUS: ACCEPTED}

BMC Infectious Diseases BMC Series

Evelyn Loo

Singapore Institute of Clinical Sciences

ح evelyn_loo@sics.a-star.edu.sgCorresponding Author

ORCiD: https://orcid.org/0000-0001-7690-3191

Amanda Zain

National University Singapore Yong Loo Lin School of Medicine

Gaik Chin Yap

National University Singapore Yong Loo Lin School of Medicine

Rikky W Purbojati

Nanyang Technological University

Daniela I Drautz-Moses

Nanyang Technological University

Yan Qing koh

Nanyang Technological University

Yap Seng Chong

National University Singapore Yong Loo Lin School of Medicine

Kok Hian Tan

KK Women's and Children's Hospital

Peter D Gluckman

Singapore Institute for Clinical Sciences

Fabian Yap

KK Women's and Children's Hospital

Johan Gunnar Eriksson

National University Singapore Yong Loo Lin School of Medicine 


\section{Elizabeth Tham}

National University Singapore Yong Loo Lin School of Medicine

lynette shek

National University Singapore Yong Loo Lin School of Medicine

Staffan kjelleberg

Nanyang Technological University

Stephan C Schuster

Nanyang Technological University

Ritu Banerjee

Vanderbilt University Medical Center

Bee Wah Lee

National University Singapore Yong Loo Lin School of Medicine

\section{DOI:}

$10.21203 / \mathrm{rs} .2 .18486 / \mathrm{v} 1$

\section{SUBJECT AREAS}

Infectious Diseases Pediatrics

\section{KEYWORDS}

Antibiotic resistance genes, resistome, infancy, birth cohort, ESBL producing Enterobacteriaceae 
Abstract

Background: The rapid spread of multidrug- resistant pathogenic bacteria is a worldwide public health concern. Given the high carriage rate of extended spectrum beta-lactamase (ESBL)- producing Enterobacteriaceae in Asia, we aimed to evaluate community prevalence and dynamics by studying the longitudinal changes in antibiotic resistance gene (ARG) profiles and prevalence of ESBLproducing $\mathrm{E}$ coli and $\mathrm{K}$. pneumoniae in the intestinal microbiome of infants participating in the Growing Up in Singapore Towards Healthy Outcomes (GUSTO) study, a longitudinal cohort study of pregnant women and their infants.

Methods: We analysed the antibiotic resistance genes profile in the first year of life among 75 infants who had stool samples collected at multiple timepoints using metagenomics.

Results: The mean number of ARGs per infant increased with age. The most common ARGs identified confer resistance to aminoglycoside, beta-lactam, macrolide and tetracycline antibiotics; all infants harboured these antibiotic resistance genes at some point in the first year of life. Few ARGs persisted throughout the first year of life. Beta-lactam resistant Escherichia coli and Klebsiella pneumoniae were detected in $4(5.3 \%)$ and $32(42.7 \%)$ of subjects respectively.

Conclusion: In this longitudinal cohort study of healthy infants living in a region with high endemic antibacterial resistance, we demonstrate that majority of the infants harboured a number of antibiotic resistance genes in their gut and showed that the infant gut resistome is diverse and dynamic over the first year of life.

Full Text

Due to technical limitations, full-text HTML conversion of this manuscript could not be completed. However, the manuscript can be downloaded and accessed as a PDF.

Figures

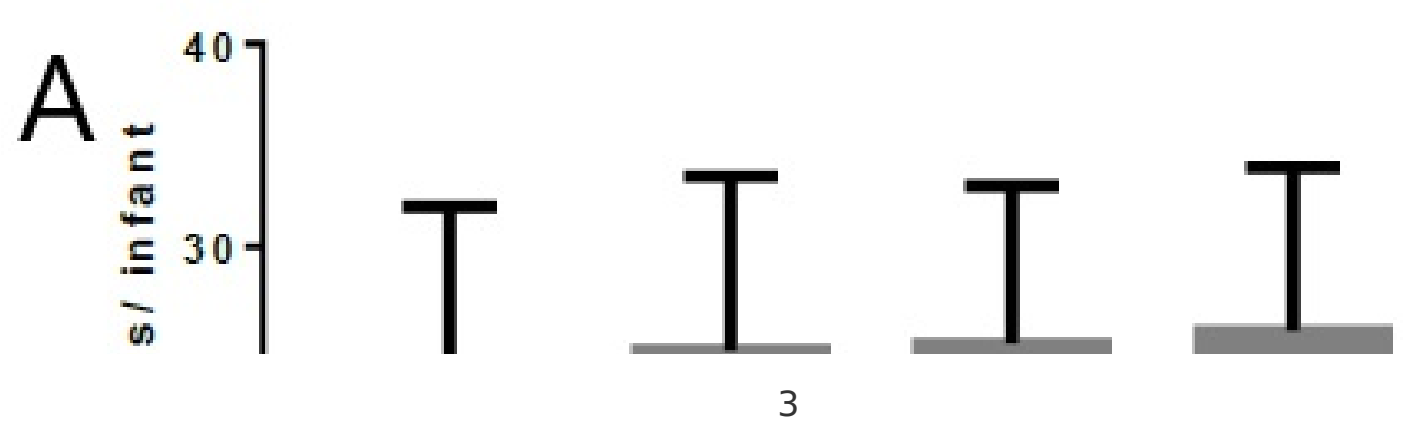




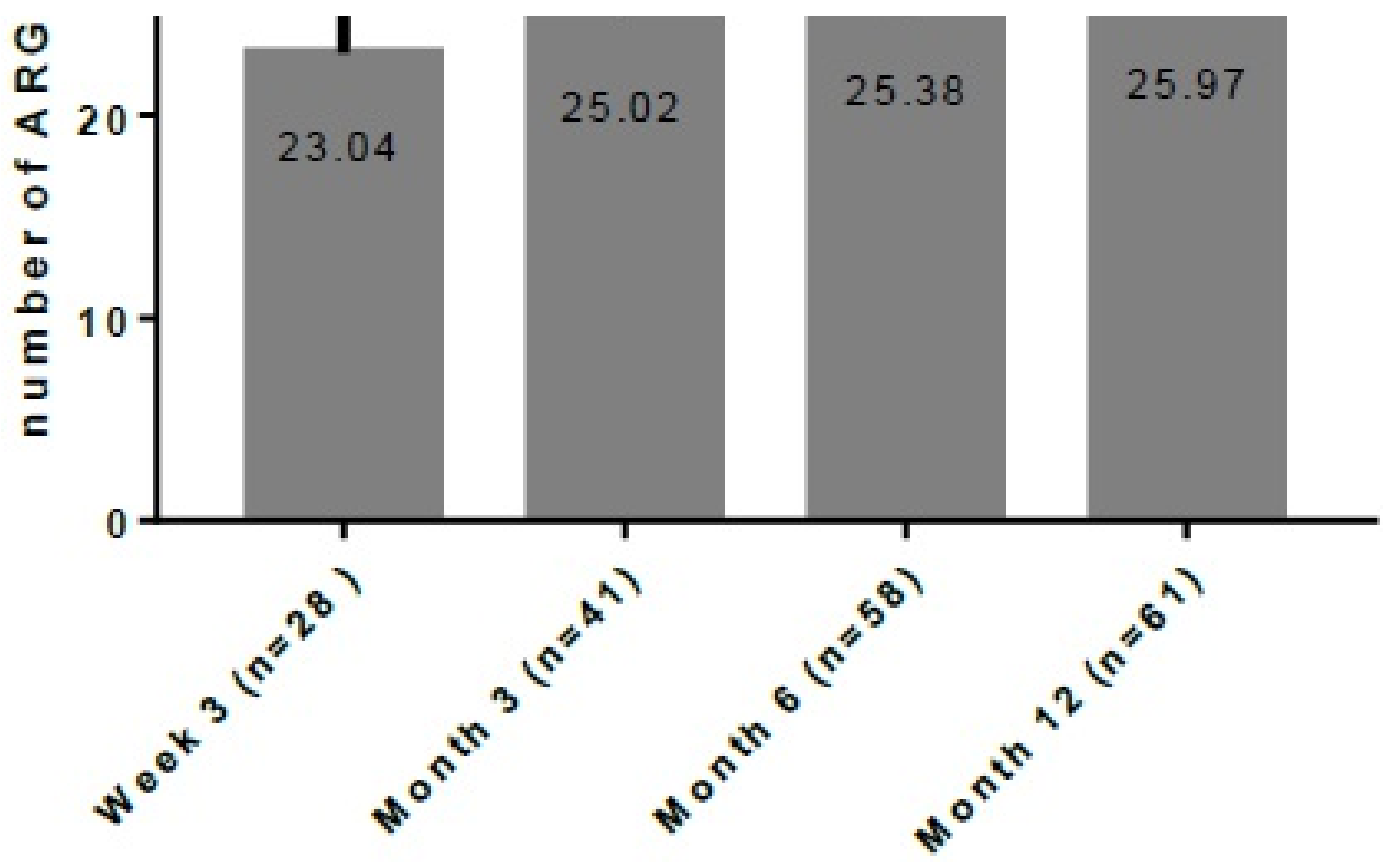

B

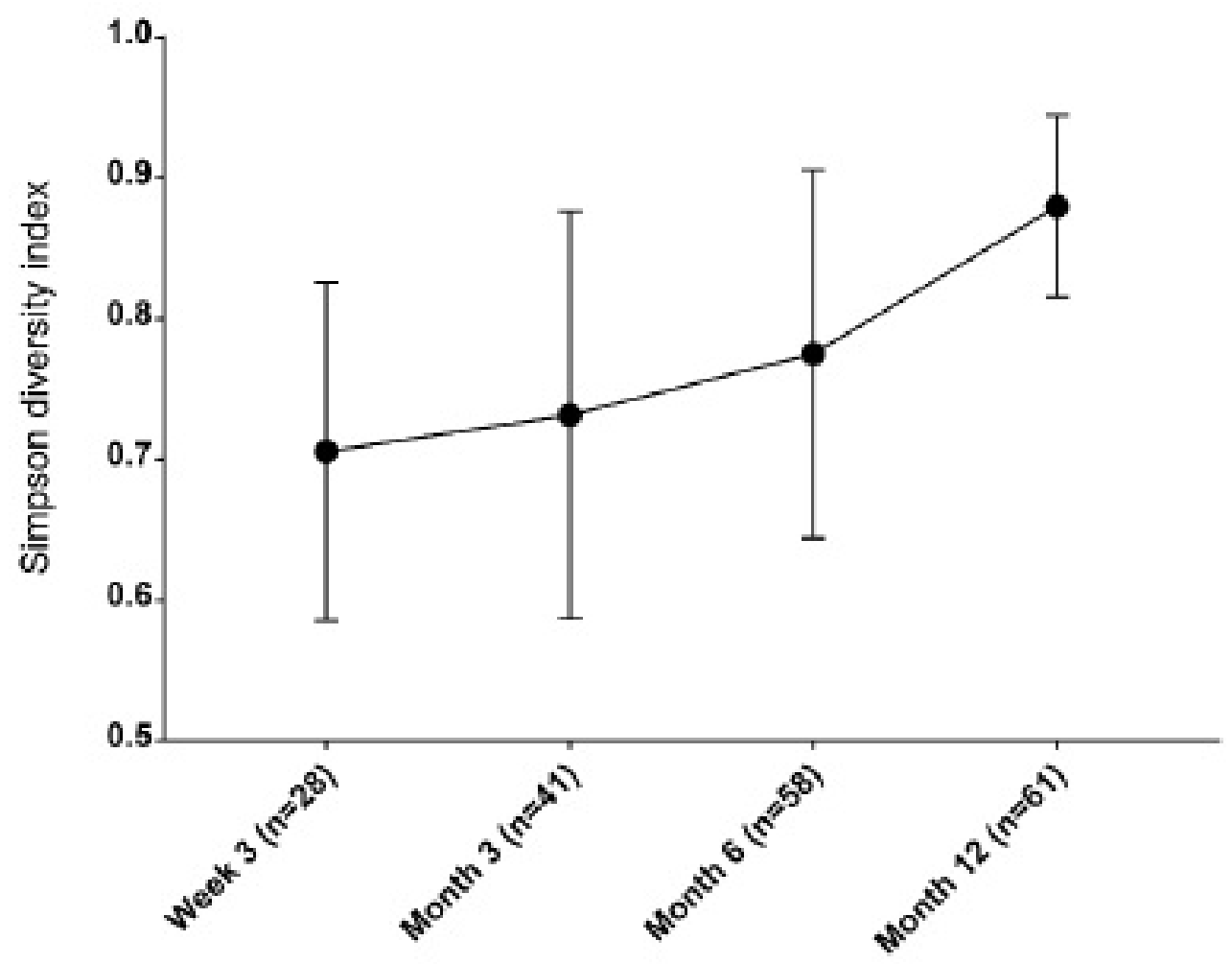

Figure 1

1a. Mean number of ARGs per infant over time. 1b Microbial diversity of stool samples over 
time as determined by Simpsons Diversity Index.

\section{Supplementary Files}

This is a list of supplementary files associated with this preprint. Click to download.

ARGpaper_Additional Data_271119.pdf 